\title{
Tidal errors and deformations in regional GPS networks
}

\author{
L. Biagi \\ Politecnico di Milano - DIIAR c/o Polo Regionale di Como, via Valleggio, 11 - 22100 Como, Italy \\ G. Pietrantonio, F. Riguzzi \\ Istituto Nazionale di Geofisica e Vulcanologia, via di Vigna Murata, 605 - 00143 Roma, Italy
}

\begin{abstract}
At present, the tidal correction model described in the IERS 2003 Conventions should be applied in the processing of permanent networks: in brief, the model involves the computation of a first order tidal correction plus some corrective terms, as functions of space and time. In the BERNESE software, one of the main scientific softwares for GPS network processing, the IERS tidal model is implemented; in July 2004, a bug in the tidal correction routine was identified by one of the paper authors and corrected by the BERNESE staff: the error concerned a second order term, related to the semidiurnal tidal signal.

An experimental analysis on the error effects in the results stemming from processing of regional networks has been carried out. A network of 8 European permanent GPS stations has been selected: the network choice ensures heterogeneity in the length and the orientation of the baselines. One year of data has been considered and two elaborations of the data have been performed using the BERNESE 4.2 SW, by applying the same processing strategy and, respectively, the wrong (old) and the right (corrected) tidal correction routine. The differences in the network results and the deformations caused by the error are discussed.
\end{abstract}

Keywords. GPS permanent networks, BERNESE SW, Deformation control, Tidal modeling error

\section{Introduction}

It is well known that every celestial body exerts a gravitational attraction on the points of the Earth; a gravitational potential is usually related to the attraction. Attraction and potential are functions of the distance between the body and the point: so, they vary both inside the Earth and on its surface.

The tidal attraction of a celestial body $\mathrm{B}$ in a point $\mathrm{P}$ of the Earth is defined as the difference of the $\mathrm{B}$ gravitational attraction between the point itself and the Earth mass center (EMC).

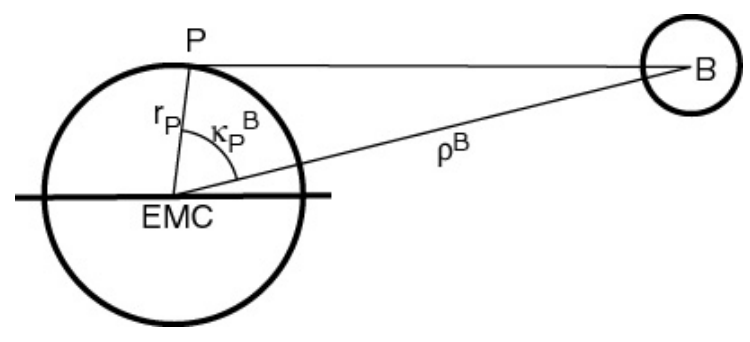

Fig. 1 The geometry of tidal potential

According to (Vanicek, 1980, Fig. 1), the related potential is:

$$
\mathrm{W}_{\mathrm{E}}^{B}(\mathrm{P})=\frac{\mathrm{G} M^{\mathrm{B}}}{\rho^{B}} \sum_{\mathrm{n}=2}^{\infty}\left(\frac{\mathrm{r}_{\mathrm{p}}}{\rho^{B}}\right)^{\mathrm{n}} \mathrm{P}_{\mathrm{n}}\left(\cos \kappa_{P}^{B}\right)
$$

where: $\mathrm{G}$ is the universal gravitational constant, $M^{B}$ is the body mass, $\rho^{B}$ is the distance between the body and the EMC, $r_{p}$ is the distance between the point $\mathrm{P}$ and the EMC, $\kappa_{P}^{B}$ is the geocentric angle between $\mathrm{B}$ and $\mathrm{P}$. It is assumed that, for each geodetic and geodynamic application, only Moon and Sun tidal attractions are significant.

In the time domain the tidal attraction shows typical bands centered on infinite, monthly, diurnal and semidiurnal periods.

Tidal attractions cause periodic deformations of the Earth's shape; obviously, in the analysis of time series of geodetic observations (VLBI, SLR, GPS, ...) finalized to the estimation of positions and long term displacements on the Earth's surface, the tidal deformation on each single epoch position estimate needs to be removed: in other terms, an accurate model of tidal deformations has to be available to process raw geodetic observations. The response of the Earth's shape to the tidal attraction depends on Love and Shida numbers. They express the actual displacement in a point as the ratio between the response of an ideally rigid and an ideally fluid 
Earth. The Love number is related to the radial (vertical) displacement in the point, while The Shida number is related to the tangential (horizontal) displacements. Due to the Earth's anelasticity, both Love and Shida numbers are complex functions both of the point position and of the tidal signal frequency.

In the last decades a lot of effort has been invested to properly model the tidal deformation: among the others, we mention the works of (Wahr, 1982, Mathews et al., 1995).

At present, the suggested formula for the tidal effects modeling on the Earth's surface is described in IERS conventions 2003 (McCarthy and Petit, 2004). Without entering into the details, the numerical tidal modeling is performed in four steps:

0 . zero order estimate, taking into account the latitude dependences of Love and Shida numbers;

1. corrections for out of phase dependences of Love and Shida numbers on the diurnal and semidiurnal frequencies of the tidal signal;

2. final corrections for the Love number dependences on the diurnal and long period frequencies;

3. computation of the permanent tide (not applied for conventionally tide free coordinates) and the polar motion tidal effects.

Several numerical routines have been implemented according to the IERS conventions: among them, the FORTRAN routine of Veronique Dehant is freely available on the website $\mathrm{ftp} / / /$ omaftp.oma.be/dist/astro/dehant/IERS; in the next months, a $\mathrm{C}$ translation of the Dehant routine will be freely available on the website http://geomatica.como.polimi.it.

The moon-sun induced Earth's tide can reach values up to some decimeters; its time series show the same frequency bands of the tidal attraction. As an example, in Fig. 2 the tidal effects for the Matera IGS GPS permanent station are shown; in Table 1 the relevant statistics are summarized.
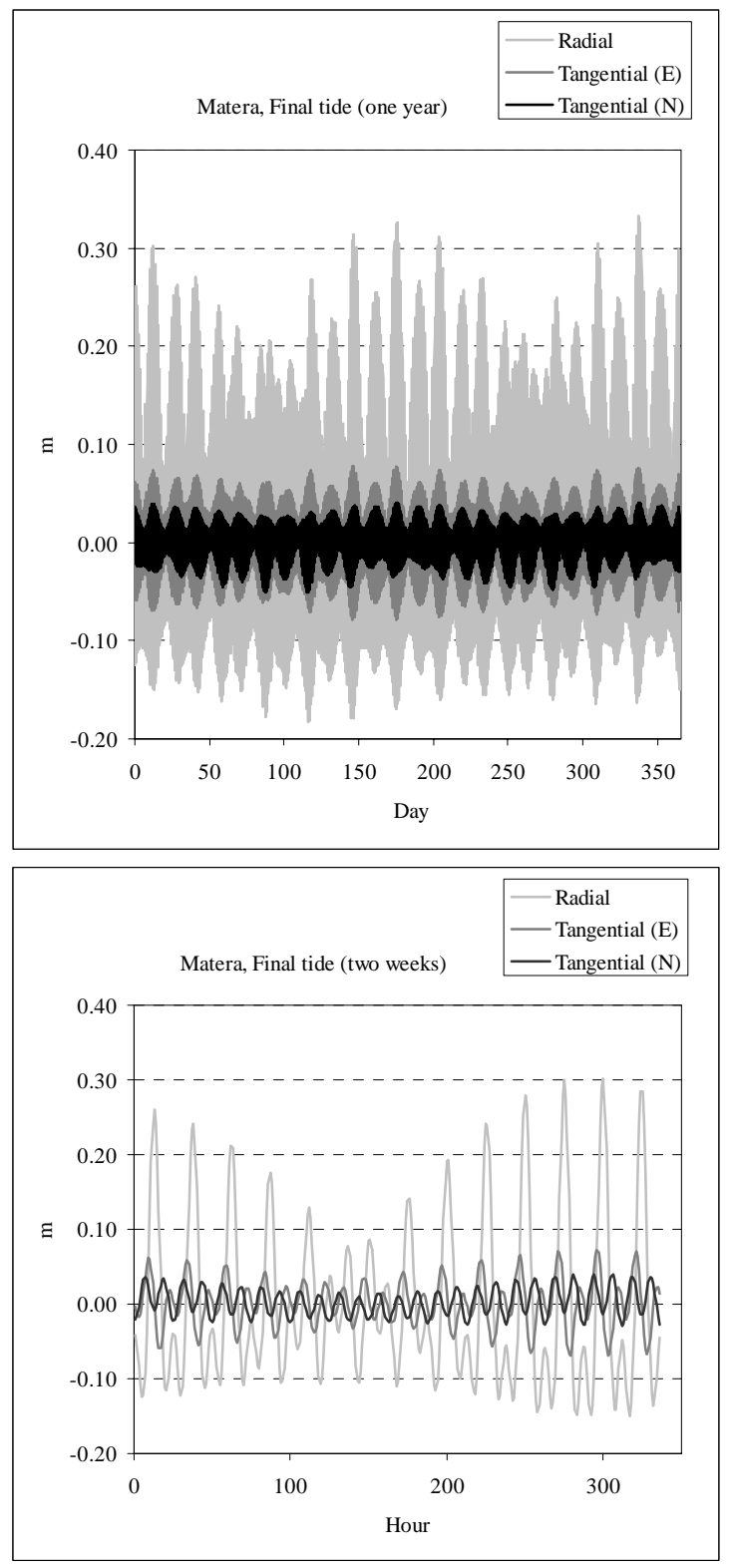

Fig. 2 An example of tidal effect.

Matera IGS station. Top: one year (from July, $1^{\text {st }}, 2003$ to June, $30^{\text {th }}$, 2004) of hourly tidal corrections. Bottom: a detail on the first two weeks.

\section{The tidal modeling in GPS data adjustment}

In the following we will shortly summarize the tidal effect, and the way to model it, on GPS observations, either code or phase. To do that, we'll consider only the distance term in the GPS observation equation, disregarding the atmospheric delays, clock errors, internal delays and integer 
ambiguities: for a complete description of them see for example (Teunissen, Kleusberg, 1998). Moreover, in the following we do not consider (assuming as already corrected for) the offsets of the satellite/receiver antenna phase centers with respect to the satellite mass center/ground station positions.

The GPS “distance” observation from a receiver $\mathrm{R}$ to a satellite $\mathrm{S}$ at a given epoch $\mathrm{t} \rho_{R}^{S}(t)$ can be written as

$\rho_{R}^{S}(t)=\left\|\mathbf{x}^{S}\left(t-\tau_{R}^{S}\right)-\mathbf{x}_{R}(t)\right\|$

where $\mathbf{x}^{S}\left(t-\tau_{R}^{S}\right)$ is the satellite mass center position at the emission epoch $t-\tau_{R}^{S}, \mathbf{x}_{R}(t)$ is the position of the station at the reception epoch $t$. Taking into account the tide, we can write:

$\mathbf{x}_{R}(t)=\mathbf{x}_{0, R}(t)+\delta \mathbf{x}_{T R}\left(\mathbf{x}_{0, R}, t\right)$

where $\mathbf{x}_{0, R}(t)$ is the "tide free" position of the station, $\delta \mathbf{x}_{T R}\left(\mathbf{x}_{0, R}, t\right)$ is the tidal effect (displacement) in the station at the reception epoch.

The distance observation equation can be linearized with respect to the tidal effect:

$$
\begin{aligned}
\rho_{R}^{S}(t) & \cong\left\|\mathbf{x}^{S}\left(t-\tau_{R}^{S}\right)-\mathbf{x}_{0, R}(t)\right\| \\
& +\mathbf{e}_{R}^{S}(t) \cdot \boldsymbol{\delta} \mathbf{x}_{T R}\left(\mathbf{x}_{0, R}, t\right)
\end{aligned}
$$

where $\mathbf{e}_{R}^{S}(t)$ is the unitary vector directed from the satellite to the station.

Given approximate values for the station position, $\tilde{\mathbf{x}}_{0, R}$, given a tidal (e.g. the IERS2003) model, we can compute the tidal correction to the observation:

$$
\delta \rho_{T R}^{S}(t)=\mathbf{e}_{R}^{S}(t) \cdot \delta \mathbf{x}_{T R}\left(\mathbf{x}_{0, R}, t\right)
$$

Next, we can compute a tide free distance observation by subtracting the tidal correction from the original observation:

$$
\begin{aligned}
\rho_{0, R}^{S}(t) & \cong \rho_{R}^{S}(t)-\delta \rho_{T R}^{S}(t) \\
& =\left\|\mathbf{x}^{S}\left(t-\tau_{R}^{S}\right)-\mathbf{x}_{0, R}(t)\right\|
\end{aligned}
$$

Finally, a set of tide free observations can be adjusted to estimate a tide free position of the station.

In GPS network adjustment, some softwares construct differences between the raw observations from two receivers to one or two satellites in order to estimate the network coordinates; particularly, the single differences between two receivers are given by the

$$
\rho_{R 1, R 2}^{S}(t)=\rho_{R 1}^{S}(t)-\rho_{R 2}^{S}(t)
$$

Double differences from two receivers to two satellites are given by the

$$
\rho_{R 1, R 2}^{S 1, S 2}(t)=\rho_{R 1, R 2}^{S 1}(t)-\rho_{R 1, R 2}^{S 2}(t)
$$

The corresponding differenced tidal corrections (dtc) are given, respectively, by the:

$$
\delta \rho_{T R 1, R 2}^{S}(t)=\delta \rho_{T R 1}^{S}(t)-\delta \rho_{T R 2}^{S}(t)
$$

and by the

$$
\delta \rho_{T R 1, R 2}^{S 1, S 2}(t)=\delta \rho_{T R 1, R 2}^{S 1}(t)-\delta \rho_{T R 1, R 2}^{S 2}(t)
$$

To compute the tide free single/double differences, two ways are possible:
1) to compute the tide free raw observations before differencing them;
2) to difference the raw observations and then to compute and subtract the dtc.

Table 1. Statistics of tidal corrections.

Matera IGS PS; one year (from July, $1^{\text {st }}, 2003$ to June, $30^{\text {th }}$, 2004) of hourly tidal corrections: 8760 data points. E: mean value; $\sigma$ : RMS of data; M: maximum; m: minimum. S0: step 0 estimates; S1: step 1 corrections; S2: step 2 corrections; PM: polar motion effect; PT: permanent tide effect; F: final estimate.

\begin{tabular}{|c|c|c|c|c|c|c|c|c|c|c|c|c|}
\hline & \multicolumn{4}{|c|}{ Radial (mm) } & \multicolumn{3}{|c|}{ Tangential, East comp. (mm) } & \multicolumn{3}{|c|}{ Tangential, North comp. (mm) } \\
\hline & $\mathrm{E}$ & $\sigma$ & $\mathrm{m}$ & $\mathrm{M}$ & $\mathrm{E}$ & $\sigma$ & $\mathrm{m}$ & $\mathrm{M}$ & $\mathrm{E}$ & $\sigma$ & $\mathrm{m}$ & $\mathrm{M}$ \\
\hline S0 & -15 & 108 & -187 & 331 & 0 & 29 & -78 & 77 & -23 & 17 & -77 & 16 \\
\hline S1 & $<1$ & $<1$ & -1 & 1 & $<1$ & $<1$ & -1 & 1 & $<1$ & $<1$ & 1 & 1 \\
\hline S2 & $<1$ & 9 & -15 & 16 & $<1$ & $<1$ & -1 & 1 & $<1$ & $<1$ & 0 & 1 \\
\hline PM & $<1$ & 4 & -7 & 6 & $<1$ & $<1$ & -1 & 1 & 0 & 0 & 0 & 0 \\
\hline PT & 16 & 0 & 16 & 16 & 0 & 0 & 0 & 0 & 25 & 0 & 25 & 25 \\
\hline F & 0 & 102 & -183 & 332 & 0 & 29 & -79 & 78 & 0 & 17 & -51 & 41 \\
\hline
\end{tabular}




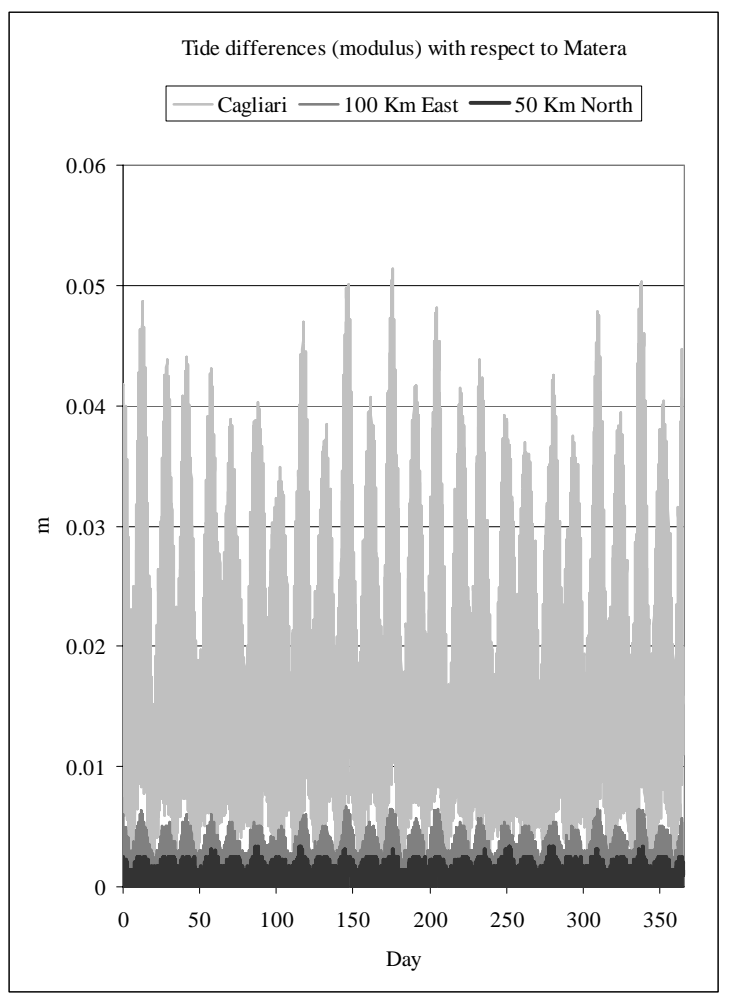

Fig. 3 An example of difference between tidal corrections for two stations.

One year (from July, $1^{\text {st }}, 2003$ to June, $30^{\text {th }}, 2004$ ) of hourly tidal corrections. The plotted time series contain the modulus of the vector of the tidal correction differences. Reference station: Matera IGS PS. Second station: Baseline 1: $50 \mathrm{~km}$ toward North, max difference $3 \mathrm{~mm}$; B2: $100 \mathrm{~km}$ toward East, max difference $7 \mathrm{~mm}$; B3 Cagliari IGS PS (680 km), max difference $5 \mathrm{~cm}$.

Both the approaches provide the same final results: the choice is purely a numerical matter. A last remark is worth: the dtc depend both on the observation geometry (absolute and relative positions of the stations and the satellites) and on the difference between the tidal displacements of the stations. In very local networks (baseline lengths smaller than $20 \mathrm{Km}$ ), tidal differences are always negligible at the $\mathrm{mm}$ level; on baselines between 20 and $100 \mathrm{Km}$, particularly if oriented in East-West direction, the differences can reach the $\mathrm{cm}$; on regional networks, they are never negligible. An example of tidal correction differences between two stations is given in Fig. 3.

\section{The tidal modeling bug in BERNESE GPS SW}

In the BERNESE SW (Beutler et al., 2000, Hugentobler et al., 2005) the tidal modeling is performed, according the IERS Conventions, by the subroutines tide96 for the 4.2 release and tide2000 for the 5.0 release. A bug in the tidal modeling was discovered by one of the authors (Biagi, personal email to Urs Hugentobler, 2004), and corrected by the AIUB staff (BSWMail 190, 2004). The bug concerned the step 2 computation; particularly, the correction to the step 1 estimate of the radial component of the tidal effect was affected.

In the following, the errors introduced in the time series by the tidal modeling error are investigated. In this section, at first the tidal modeling errors on a single station (absolute errors) are presented; in the following, the differences between the tidal modeling errors for two stations (relative errors) are discussed. In the next section, errors in position estimates caused by the tidal modeling errors in the GPS data adjustment for a network are shown.

As an example, the Matera and the Cagliari IGS permanent stations have been chosen: their distance is about $680 \mathrm{Km}$ (Fig. 6, Table 4).

In order to investigate long term periodicity of the errors, two years have been analyzed, from January, $1^{\text {st }}, 2003$ to December, 31, 2004. Adopting a computation interval of one hour, both the right and the wrong tidal corrections have been computed: absolute tidal errors for both stations have been derived by subtracting the right from the wrong tidal correction. Absolute errors are shown in Fig. 4 for Matera; the relevant statistics are summarized in Table 2 for both stations. The absolute tidal errors show two clear periods: the first diurnal (Fig. 4, bottom), the second annual (Fig. 4, top), both with an amplitude of about 25-30 $\mathrm{mm}$. The errors have zero mean, with maxima and minima of about $30 \mathrm{~mm}$.

Table 2. BERNESE absolute tidal errors on radial component.

Two years (from January, $1^{\text {st }}, 2003$ to December, $31^{\text {st }}, 2004$ ) of differences between wrong and right tidal corrections: 17520 epochs. E: mean error; $\sigma$ : RMS of the errors; m: minimum; M: maximum.

\begin{tabular}{|c|c|c|c|c|}
\hline & $\mathrm{E}(\mathrm{mm})$ & $\sigma(\mathrm{mm})$ & $\mathrm{m}(\mathrm{mm})$ & $\mathrm{M}(\mathrm{mm})$ \\
\hline Matera & 0 & 14 & -30 & 30 \\
\hline Cagliari & 0 & 13 & -29 & 30 \\
\hline
\end{tabular}



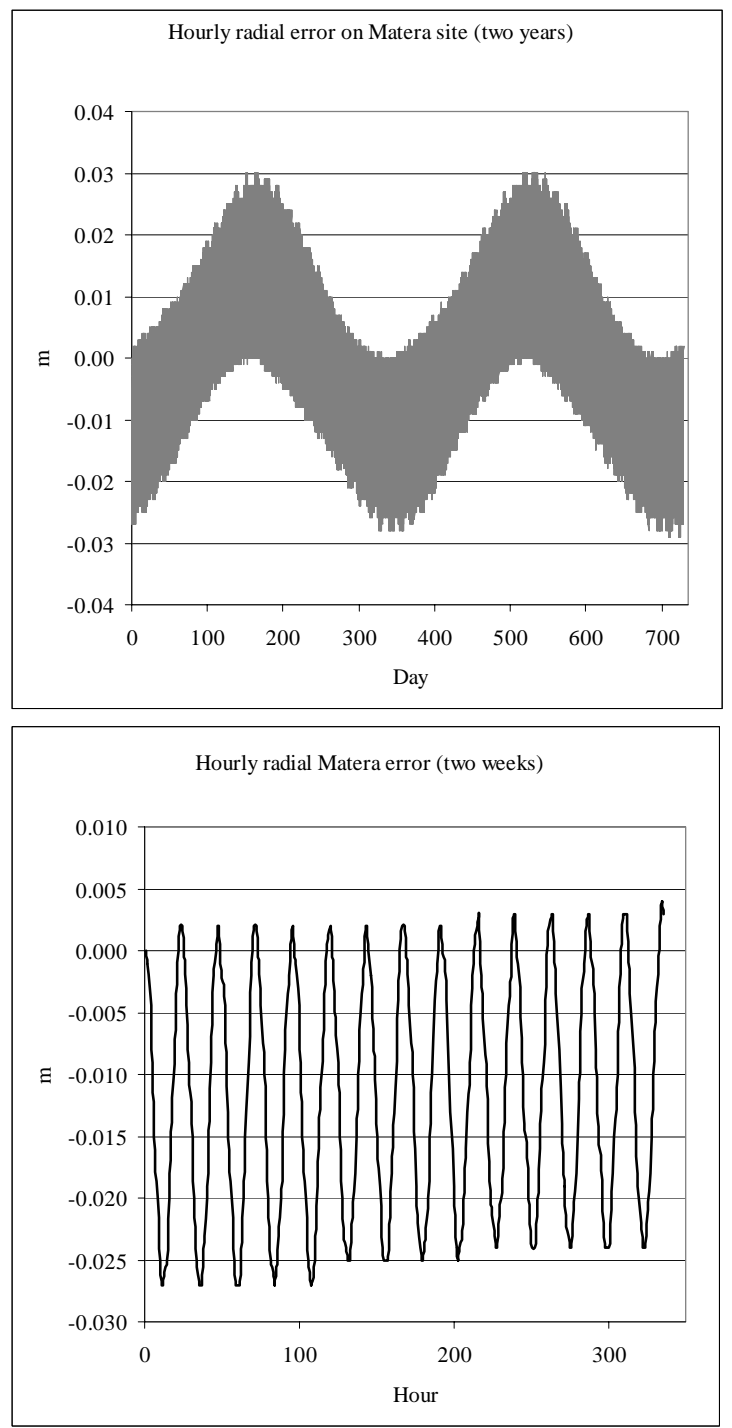

Fig. 4 (up). BERNESE absolute tidal errors on radial component. Matera IGS station. Top: two years (from January, $1^{\text {st }}, 2003$ to December, $\left.31^{\text {st }}, 2004\right)$ of differences between right and wrong tidal corrections. Bottom: detail of the first two weeks. Not the same scale for the graphs.

Fig. 5 (on the right). Relative tidal errors between Matera and Cagliari IGS stations (distance $680 \mathrm{~km}$ ).

The three components of the relative tidal error vectors have been converted from cartesian ITRF2000 components to local (with respect to Cagliari position) North, East, Up. Two years (from January, $1^{\text {st }}, 2003$ to December, 31 $1^{\text {st }}, 2004$ ) of data. Top: hourly tidal error differences. Center: mean daily error differences. Bottom: RMS of the 24 hourly data for each day in the two years. Not the same scale for the three graphs.
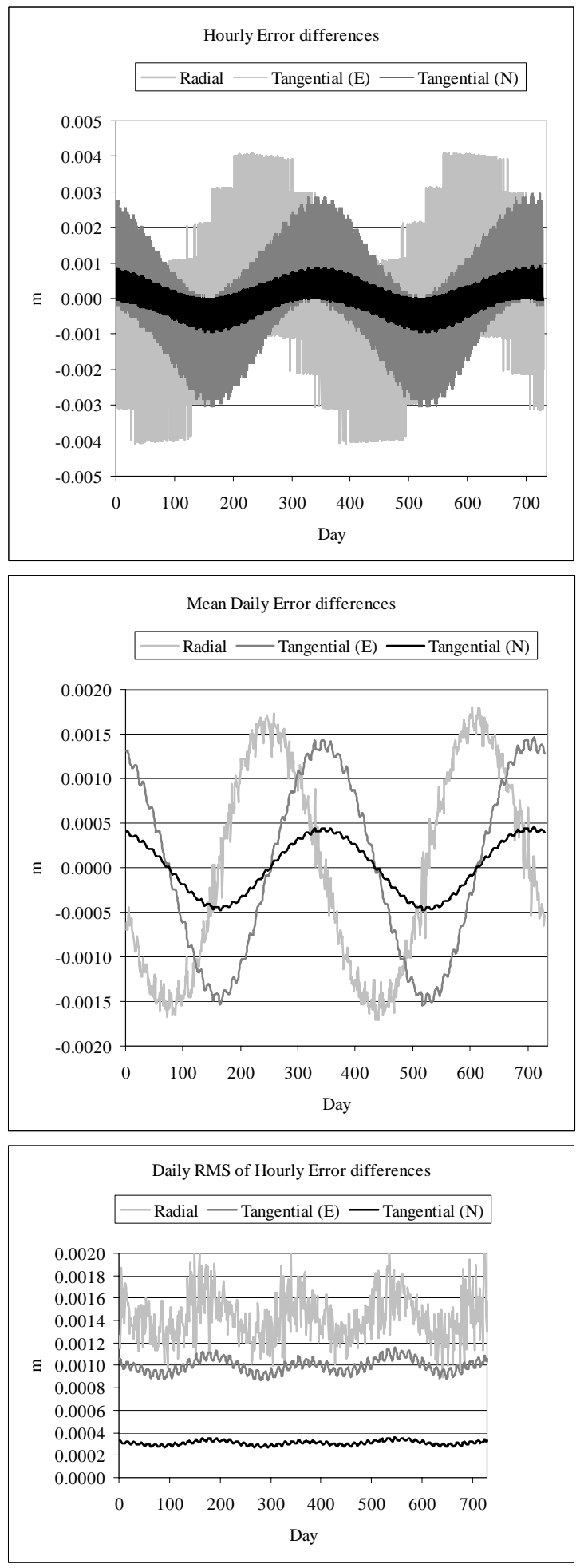
Table 3. Relative tidal errors between Matera and Cagliari IGS stations (distance $680 \mathrm{~km}$ ). Two years (from January, $1^{\text {st }}$, 2003 to December, $31^{\text {st }}$, 2004) of data. The three components of the relative tidal error vectors have been converted from cartesian ITRF2000 components to local (with respect to Cagliari position) North, East, Up. H: hourly differences. D: mean daily differences. E: mean error; $\sigma$ : RMS of the errors; m: minimum; M: maximum.

\begin{tabular}{|c|c|c|c|c|}
\hline & $\mathrm{E}(\mathrm{mm})$ & $\sigma(\mathrm{mm})$ & $\mathrm{m}(\mathrm{mm})$ & $\mathrm{M}(\mathrm{mm})$ \\
\hline $\mathrm{N}, \mathrm{H}$ & 0 & 0.4 & -0.9 & 0.9 \\
\hline E, H & 0 & 1.4 & -3.1 & 3.0 \\
\hline U, H & 0 & 1.8 & -4.1 & 4.0 \\
\hline N, D & 0 & 0.3 & -0.5 & 0.5 \\
\hline E, D & 0 & 1.0 & -1.4 & 1.5 \\
\hline U, D & 0 & 1.1 & -1.8 & 1.7 \\
\hline
\end{tabular}

In the GPS data adjustment, BERNESE SW constructs and uses double differences: so, only tidal error differences influence the final results. Moreover, typically the GPS data of a permanent network are processed on a daily basis, in order to estimate daily coordinates. So, mean daily error differences seem to be particularly interesting. Fig. 5 and Table 3 summarize the hourly and mean daily error differences between Matera and Cagliari. The error vectors have been converted from cartesian ITRF2000 components to local (with respect to Cagliari position) North, East and Up directions. The relative errors are significant at the $\mathrm{mm}$ level; their daily means are not on average null, and show a clear annual period: this may involve significant effects also on GPS daily solutions. Moreover, the greater errors are in the local Up and East directions: taking into account the orientation of the baseline between Matera and Cagliari (Fig. 6), in GPS data processing this should imply errors mainly in the estimates of heights and baseline lengths.

\section{Data set and processing}

A permanent GPS network (Fig. 6) of 8 stations has been selected ensuring heterogeneity in the length and orientation of the baselines: Matera (MATE), Cagliari (CAGL), Villafranca (VILL), Wettzell (WTZR), Roma (INGR and MOSE), Roseto degli Abruzzi (RSTO) and Perugia (UNPG).

According to the periodicity in the tidal correction errors previously evidenced, one year of data has been considered, from July, $1^{\text {st }}, 2003$ to June, $30^{\text {th }}$ ， 2004. We processed the GPS observations twice by the BPE automatic procedure of the BERNESE SW, 4.2 (Beutler et al., 2000). In both the network adjustments, the EUREF (EPN, 2002) guidelines for regional network adjustment were applied. The datum was fixed by using IGS final ephemerides, IERS C04 EOP, and by constraining the Matera coordinates to their ITRF2000 estimates, daily propagated. The main characteristics of the implicitly defined baselines between Matera and the others stations are summarized in Table 4.

In the first processing, the wrong (old) tidal model was applied to correct GPS observations; in the second processing the right (corrected) tidal correction was applied. The differences (tidal error effects) between the results are discussed.

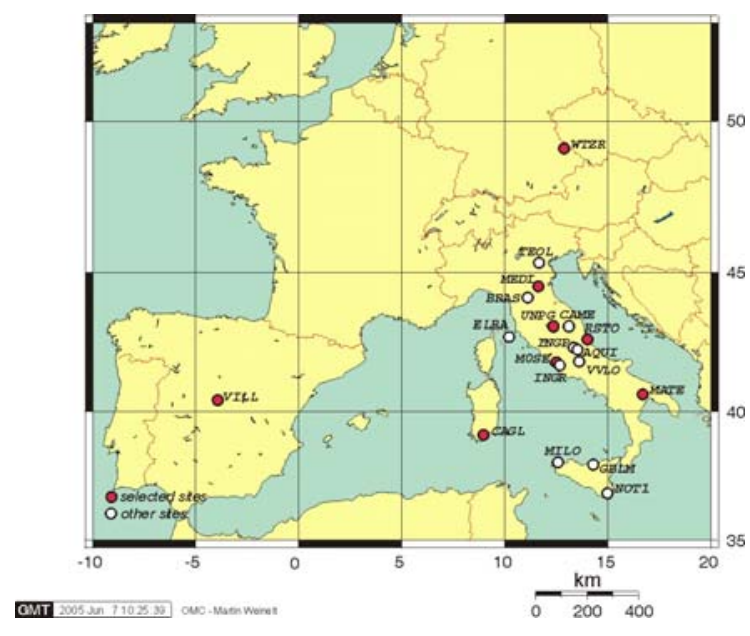

Fig. 6 The test network (7 baselines considered, from 320 (MATE-RSTO) to $1740 \mathrm{Km}$ (MATE-VILL) long.

Table 4. Main characteristics of the network baselines. L: baseline length; A: baseline azimuth, clockwise from North direction; Dh: height difference between the stations.

\begin{tabular}{|c|c|c|c|}
\hline Matera to & L (km) & A $\left(^{\circ}\right)$ & Dh (m) \\
\hline RSTO & 317 & 306 & 433 \\
\hline MOSE & 379 & 286 & 415 \\
\hline UNPG & 453 & 300 & 185 \\
\hline MEDI & 597 & 307 & 486 \\
\hline CAGL & 682 & 259 & 297 \\
\hline WTZR & 990 & 336 & 124 \\
\hline VILL & 1741 & 270 & 112 \\
\hline
\end{tabular}

At first, we focus our analysis on the tidal error effects on the coordinate estimates. The differences between the estimated coordinates show a clear annual signal, as anticipated from the analysis of the 
mean daily errors. The magnitudes of the errors linearly depends on the baseline length, and are of the order of some parts per billion. Typically, the horizontal errors are oriented along the baseline direction: this fact should involve a scale effect on the baseline length estimate, that will be analyzed at the end of the section; for the majority of the stations, horizontal and vertical errors are comparable: an exception is provided by the Wettzell station, for which the vertical errors are significantly smaller. In Fig. 7 and Table 5 the results for some stations, representative of the different classes of distances from Matera, are shown.

It has to be mentioned that the tidal errors are negligible with respect to the dispersion of the daily results: an example (Medicina estimates) is given by Fig. 8; moreover, the mean value of the errors over one year of results doesn't significantly differ from zero. Nevertheless, due to the periodicity of the tidal errors, their effect isn't expected to vanish in coordinates and velocities estimated from time series shorter than one year.

As regards the baseline lengths (Fig. 8, bottom; Fig. 9; Table 6), the same considerations can be repeated: the differences are not significant with respect to the dispersion of the daily results; the yearly estimated trends of the baseline lengths in the two cases (right and wrong tidal corrections) do not change significantly (Fisher test on differences between estimated initial length and length time derivative, with significance $\alpha$ both $=5 \%$ and $=1 \%$ ); however, tidal error effects are highly correlated in time and show a clear annual signal, with a magnitude of $5 \times 10^{-9}$ of the baseline length.

To conclude, it's worth noting that a time shift of about 2.5 months exists between the error signals in the height and in the baseline length estimates.

\section{Conclusions}

A bug has been discovered in the BERNESE routine for tidal modeling and correction. In the daily adjustment of a regional GPS network, the bug introduced errors in height and scale estimates that were not significant with respect to the dispersions of the results; moreover, on an annual basis, they had zero mean.

However, the errors show a high correlation in time; particularly, a clear annual signal is present, both in the height estimates and in the scale of GPS networks, that reaches $5 \times 10^{-9}$.

\section{Acknowledgements}

We wish to thank the Italian Space Agency and the "GPS networks and Geodesy" group of INGV for providing GPS observations. We received useful suggestions by M. Crespi. L. Biagi's work has been supported in the framework of the "Satellite Positioning Services for the e-government" Italian PRIN (MIUR founded) 2004 project.

Table 5. Tidal error effects on BERNESE daily estimates. One year (from July, $1^{\text {st }}$, 2003 to June, $30^{\text {th }}, 2004$ ) of data. Reference station: Matera IGS station. V-: Villafranca IGS station; W-: Wettzel IGS station; M-: Medicina IGS station. R- Università di Roma La Sapienza station.

Dh, DN, DE: vertical, local North and East components of the errors. E: mean error; $\sigma$ : RMS of the errors; $m$ : minimum; M: maximum.

\begin{tabular}{|c|c|c|c|c|}
\hline & E (mm) & $\sigma(\mathrm{mm})$ & $\mathrm{m}(\mathrm{mm})$ & $\mathrm{M}(\mathrm{mm})$ \\
\hline V-Dh & 0.4 & 3.8 & -9.6 & 8.9 \\
\hline V-DN & 0.1 & 1.0 & -3.9 & 3.7 \\
\hline V-DE & 0.4 & 4.7 & -12.2 & 8.7 \\
\hline W-Dh & 0.0 & 1.0 & -2.7 & 3.3 \\
\hline W-DN & 0.0 & 2.0 & -3.9 & 3.7 \\
\hline W-DE & 0.0 & 1.1 & -2.5 & 3.1 \\
\hline M-Dh & 0.1 & 0.9 & -2.3 & 1.8 \\
\hline M-DN & 0.0 & 0.9 & -1.8 & 1.8 \\
\hline M-DE & 0.1 & 0.9 & -1.6 & 1.5 \\
\hline R-Dh & 0.1 & 0.9 & -2.2 & 5.0 \\
\hline R-DN & -0.1 & 0.3 & -1.2 & 0.5 \\
\hline R-DE & 0.0 & 0.7 & -1.3 & 1.7 \\
\hline
\end{tabular}

Table 6. Tidal error effects on BERNESE daily baseline length estimates. One year (from July, $1^{\text {st }}, 2003$ to June, $30^{\text {th }}$, 2004) of data. Reference station: Matera IGS station. E: mean error; $\sigma$ : RMS of the errors; m: minimum; M: maximum.

\begin{tabular}{|c|r|r|r|r|}
\hline & $\mathrm{E}(\mathrm{mm})$ & $\sigma(\mathrm{mm})$ & $\mathrm{m}(\mathrm{mm})$ & $\mathrm{M}(\mathrm{mm})$ \\
\hline RSTO & -0.1 & 0.7 & -1.8 & 2.4 \\
\hline MOSE & -0.1 & 0.8 & -2.0 & 2.3 \\
\hline UNPG & 0.1 & 1.0 & -2.2 & 2.8 \\
\hline MEDI & 0.0 & 1.3 & -2.6 & 2.6 \\
\hline CAGL & 0.6 & 1.2 & -2.3 & 3.5 \\
\hline WTZR & -0.1 & 2.2 & -5.5 & 4.5 \\
\hline VILL & -0.4 & 3.8 & -7.0 & 10.5 \\
\hline
\end{tabular}



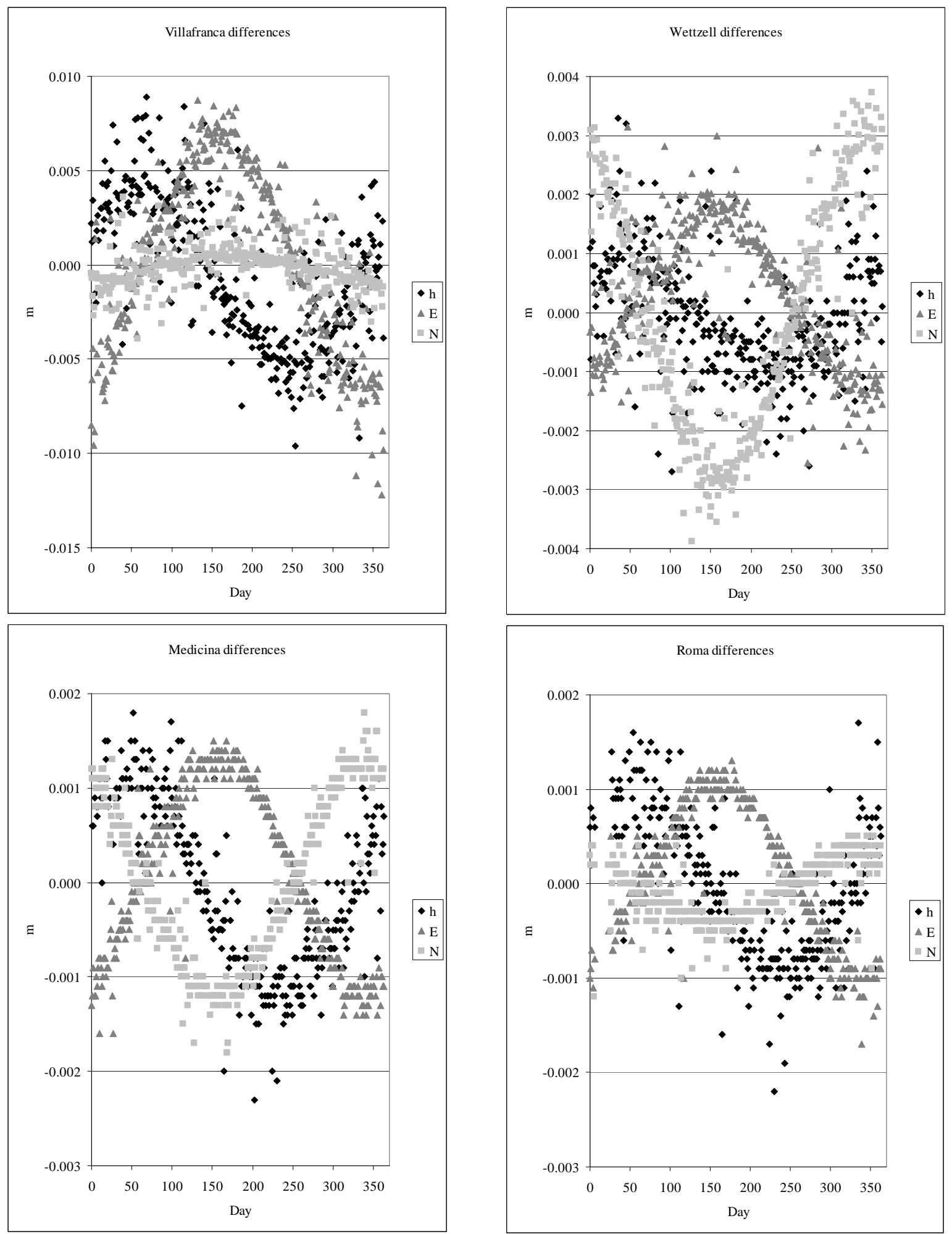

Fig. 7 Tidal error effects on BERNESE daily estimates. One year (from July, $1^{\text {st }}, 2003$ to June, 30 ${ }^{\text {th }}$, 2004) of data. Reference station: Matera IGS station. Top: Villafranca IGS station; Wettzel IGS station; bottom: Medicina IGS station; Università di Roma La Sapienza station. Not the same scale for the graphs. 

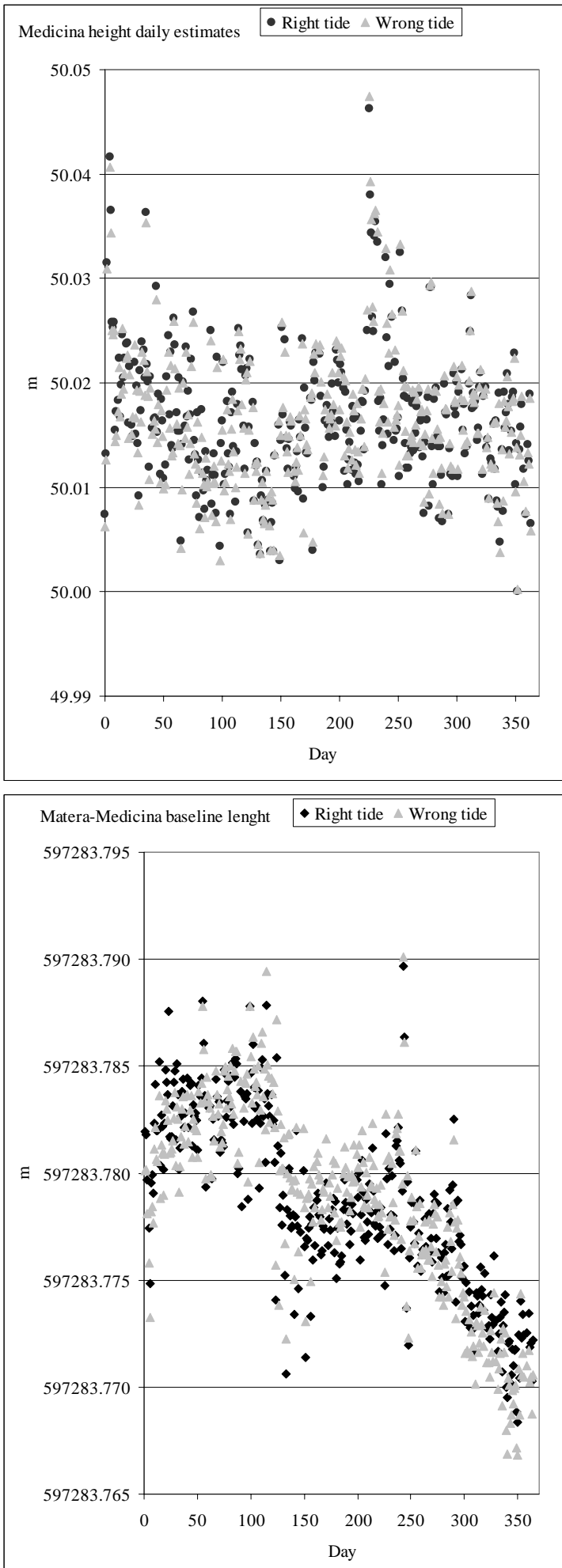

Fig. 8. Medicina IGS station daily estimates. One year (from July, $1^{\text {st }}, 2003$ to June, $30^{\text {th }}$, 2004) of data. Reference station: Matera IGS station. Top: height estimates (mean height estimate: $50.015 \mathrm{~m}$; RMS: $7 \mathrm{~mm}$; min. value: $50 \mathrm{~m}$; max. value: $50.046 \mathrm{~m})$. Bottom: baseline length estimates.

Trend: $\quad l(t)=l_{0}+v \cdot t, v=-1.1 \mathrm{~cm} / y, \sigma_{v}=0.6 \mathrm{~mm} / y$ Not the same scale for the graphs.

\section{References}

Beutler, G., Brockmann, E., Dach, R., Fridez, P., Gurtner, W., Hugentobler, U., Johnson, J., Mervant, L., Rothacher, M., Schaer, S., Springer, T., Weber, R. (2000). Bernese Software 4.2. Astronomical Institute, University of Berne.

EPN (2002). Guidelines for EPN analysis centres. Document and related mails on-line: http://www.epncb.oma.be/

Hugentobler, U., Dach, R., Fridez, P. (2005). Bernese GPS Software 5.0, Draft. Astronomical Institute, University of Berne.

Hugentobler, U., BSWMail 190 (2004), on-line: http://www.aiub.unibe.ch/download/bswmail/

Mathews, P. M., Buffett, B. A., Shapiro, I. I. (1995). Love numbers for a rotating spheroidal Earth: new definitions and numerical values. Geophys. Res. Lett., 22, pp 579582.

McCarthy, D. D., Petit, G. eds. (2004). IERS Conventions (2003). IERS Technical Note No. 32, Verlag des Bundesamts für Kartographie und Geodäsie, Frankfurt am Main.

Teunissen, P. J. G., Kleusberg A., eds (1998). GPS for Geodesy, $2^{\text {nd }}$ edition. Springer Verlag Berlin.\#\#

Vanicek, P. (1980). Tidal Corrections to Geodetic Quantities. NOAA Technical Report, NOS 83 NGS 14, US Department of Commerce, Rockville, Maryland.

Wahr, J. M. (1982). Computing tides, mutations and tidally induced variations in the Earth rotation rate for a rotating, elliptical Earth in Geodesy and Global Geodynamics, Moritz H., Sünkel H. eds, Mitteilungen der geodätischen Institute der Technischen Universität, Folge 41, Graz.

Astronomical Institute, University of Berne AIUB FTP site: ftp.unibe.ch 


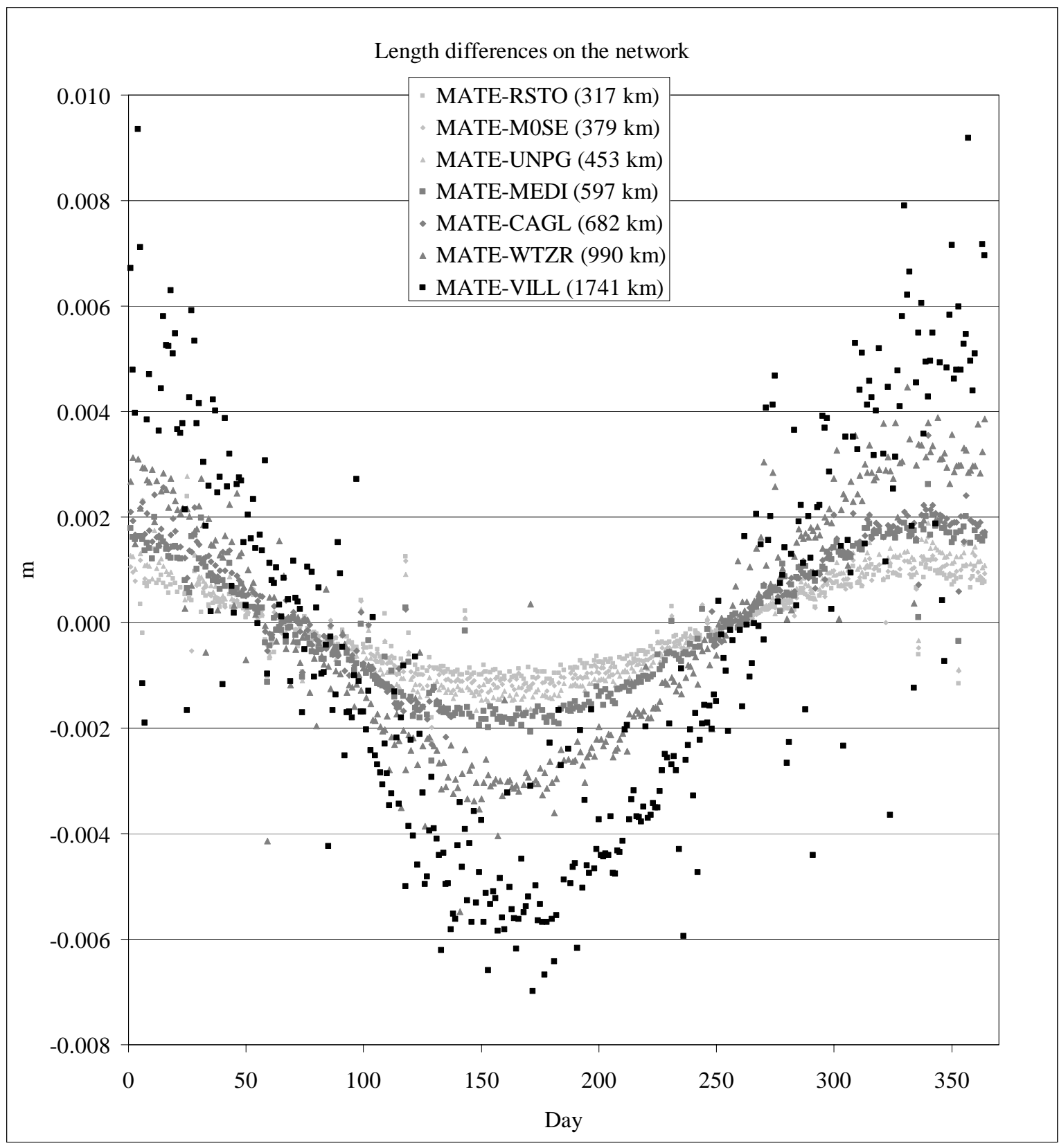

Fig. 9. Baseline lengths differences. One year (from July, $1^{\text {st }}, 2003$ to June, $30^{\text {th }}$, 2004) of data: a clear scale effect is evidenced. 